\title{
Evaluation Of Laryngeal Vascular Changes With Image1 S Enhancement System In Reference To The European Laryngological Society Guideline
}

\author{
Sibel Yıldırım${ }^{1}$, Tarık Küçük ${ }^{1}$, Zahide Büyükatalay ${ }^{1}$, Muhammet Gökmen ${ }^{1}$, MUSTAFA \\ KURSAT GOKCAN ${ }^{1}$, and Gürsel Dursun ${ }^{1}$ \\ ${ }^{1}$ Ankara University Faculty of Medicine
}

January 14, 2021

\begin{abstract}
Objectives: To assess the feasibility of using Image1 S endoscopic enhancement system for discrimination of the vascular patterns in laryngeal lesions. Design: Forty patients presenting with benign, dysplastic and malign laryngeal lesions were examined with Image1 S system. The vascular patterns were classified by a group of the authors/ according to the ELS guideline, as perpendicular or longitudinal, in all lesions. Endoscopic images of the vascular patterns are evaluated through an online survey by a group of otolaryngologists with different levels of clinical expertise. The qualitative evaluation of the vascular patterns in two groups were compared to investigate the consistency. The relationship between the vascular patterns and the pathological results was statistically analyzed. Results: Eleven patient presented with benign, 13 patients with dysplastic and 16 patients with malignant pathology. The vascular patterns were longitudinal in 9 lesions, perpendicular in 28 lesions and undetectable in 3 lesions. The relevance between the vascularization pattern and the pathological diagnosis was found to be significant $(\chi 2=20.30, \mathrm{p}<0,001)$. The qualitative evaluation of the vascular patterns by the two groups of observers was significantly close to each other $(=0.63)$. The survey also showed that spectral modes producing high contrast images were preferred by $81,9 \%$ of the participants over white light images. Conclusion: This study demonstrated the usefulness and feasibility of Image1 S endoscopic enhancement system to predict the diagnosis from vascular changes in laryngeal lesions.
\end{abstract}

Evaluation of Laryngeal Vascular Changes with Image1 S Enhancement System In Reference to the European Laryngological Society Guideline

\section{Abstract}

Objectives: To assess the feasibility of using Image1 S endoscopic enhancement system for discrimination of the vascular patterns in laryngeal lesions.

Design: Forty patients presenting with benign, dysplastic and malign laryngeal lesions were examined with Image1 S system. The vascular patterns were classified by a group of the authors/ according to the European Laryngological Society (ELS) guideline, as perpendicular or longitudinal, in all lesions. Endoscopic images of the vascular patterns are evaluated through an online survey by a group of otolaryngologists with different levels of clinical expertise. The qualitative evaluation of the vascular patterns in two groups were compared to investigate the consistency. The relationship between the vascular patterns and the pathological results was statistically analyzed.

Results: Eleven patient presented with benign, 13 patients with dysplastic and 16 patients with malignant pathology. The vascular patterns were longitudinal in 9 lesions, perpendicular in 28 lesions and undetectable in 3 lesions. The relevance between the vascularization pattern and the pathological diagnosis was found to be significant $\left(\chi^{2}=20.30, p<0,001\right)$. The qualitative evaluation of the vascular patterns by the two groups 
of observers was significantly close to each other $(?=0.63)$. The survey also showed that spectral modes producing high contrast images were preferred by $81,9 \%$ of the participants over white light images.

Conclusion: This study demonstrated the usefulness and feasibility of Image1 S endoscopic enhancement system to predict the diagnosis from vascular changes in laryngeal lesions.

Key Words: dysplasia, image enhancement systems, Image1 S, laryngeal endoscopy, longitudinal vascular pattern, perpendicular vascular pattern

\section{Key Points}

1. Image1 $\mathrm{S}$ is an effective and practical endoscopic technique for evaluation of the vascular characteristics of laryngeal lesions to predict malignancy.

2. Learning curve for endoscopic evaluation of laryngeal lesions with Image1 $\mathrm{S}$ enhancement system is irrespective of evaluator's working experience.

3. The spectral modes (Spectra A and Spectra B) were preferred over white light when examining laryngeal lesions.

\section{INTRODUCTION}

Endoscopic examination of the larynx typically focuses on the surface of the mucosa. However, finer details of the mucosal and submucosal vascular structures are possible due to the recent integration of image enhancement systems using optical tools and digital technology into conventional endoscopic systems. Inspection of mucosal pathologies by these endoscopic enhancement systems has revealed vascular characteristics of the inflammatory and the tumorigenic processes and especially the neo-angiogenesis related to carcinogenesis.

One of these enhancement systems is narrow-band imaging (NBI), which uses optically filtered light to produce high contrast images of the vasculature for endoscopic diagnosis of malignant and precancerous lesions (1). A corresponding study by the European Laryngological Society (ELS), using NBI, defined the vascularization patterns associated with laryngeal pathologies in a descriptive guideline (2). Recently, a novel image enhancement system, Image1 S, has been successfully used in different branches of medicine with the same aim; to make endoscopic images clearer and vascular network identifiable $(3,4)$. This system is based on software that uses the spectral light separations to produce images with different contrast characteristics. The spectral separation is obtained from the camera console and is enhanced by color processing algorithms.

In the present study, we attempted to evaluate the practical usefulness of the Image1 S system for the detecting the vascular patterns associated with benign, premalignant, and malignant lesions. Here, we included a group of otolaryngologists with different levels of proficiency as observers to discriminate between the vascular patterns defined by the ELS and determined the level of accuracy and consistency among the observers.

\section{MATERIALS METHODS}

The study was approved by the institutional ethics committee and performed in accordance with the good clinical practice, and applicable regulatory requirements. Informed written consents were obtained from all participants.

The study was conducted between October 2017 and October 2018 in a university hospital in the department of otorhinolaryngology. The study included 40 patients who presented with a laryngeal mass and underwent microlaryngoscopy under general anesthesia. Patients under 18 years of age were not included.

\section{Evaluation of Vascular Changes and Histological Examination}

All patients were examined intraoperatively with 0 and/or 30-degree rigid endoscopes (Karl Storz®), Germany). The vascularization patterns of the lesions were visualized using white light mode and the Spectra light modes A and/or B (Image1 S, Karl Storz, Germany). Spectra A mode was utilized to filter out red in the tissue, while intensifying the remaining colors, thereby enhancing the contrast of capillaries in the mucosa and submucosa. Spectra B mode was used to achieve a higher contrast by increasing the green and 
blue spectra (5). Side-by-side monitor views of white light and one of the spectral modes were obtained during endoscopic examination of each patient for survey analysis.

Laryngeal suspension and endoscopic examinations with white light and spectral modes were performed at the operating theater by three senior otolaryngologists (authors BK, CB, KG).

The evaluation was based on qualitative observations of the images. The qualitative data were collected by the authors through direct observation and from the participants through an electronic survey. The qualitative data were analyzed comparing and contrasting the endoscopic images and interpreting the vascular patterns as longitudinal or perpendicular in accordance with the ELS classification.

The judgment on the differentiation of the vascular patterns was made unaware of the pathological diagnosis. No consensus was reached on three cases; these were excluded from the survey.

Under endoscopic control, an incisional biopsy or an excisional biopsy was taken for histopathological examination. Pathologists specialized in head and neck lesions examined specimens, blinded to the vascular characteristics of the endoscopic images. The histopathological results were categorized into three groups as benign, malignant, and dysplastic changes. Polyps, cysts, inflammation, and Reinke's edema were classified as benign. Malignant pathology was defined as invasive squamous cell carcinoma. Dysplastic changes were classified according to the World Health Organization (WHO) grading system as low grade and high grade dysplasia (6).

The relationship between the vascular patterns and the pathological diagnosis was analyzed.

\section{Survey}

After exclusion of three lesions with undetectable vascularization patterns, online survey included 74 highdefinition digital images of 37 lesions, and the questions below:

What is the pattern of vascularization in each picture?

Which light mode reveals vasculatures better?

The survey was distributed to a group of otolaryngologists who had none or limited experience in using image enhancement systems to define laryngeal vascular changes according to the ELS classification. We preferred a heterogeneous sampling method, also known as the maximum variation sampling method, as the purposive sampling technique as it captures a wide range of experience relating to the examination of the laryngeal lesions. The participants were selected from the whole population of otolaryngology residents and fellows in Turkey; the group comprised 12 residents in the minimum second year of their training program, 8 junior fellows within 10 years after termination of the training program, and 10 seniors with more than 10 years of work experience in general otolaryngology. The survey was sent to the participants via social media groups, with brief information about the ELS classification. In every case, the participants identified the predominant type of vascular pattern on the pictures and then selected the clearest picture in the pair.

Participants classified the vasculatures as pertaining to longitudinal or perpendicular patterns, being unaware of the authors' evaluations and final pathological diagnoses. The evaluation of the images by the participants was compared to the evaluation of the authors to determine the congruence of the answers. The evaluation of the participants was correlated with their previous work experience in calendar years.

\section{Statistics}

Statistical analysis was performed using the Statistical Package for the Social Sciences version 25 software program (SPSS Inc., USA). A p value $<0.05$ was considered statistically significant. The $\chi^{2}$ (chi square) statistic and a contingency table were used to evaluate the relevance between the vascularization patterns and the pathological diagnosis.

The congruence between the two groups of evaluators (the authors and the participants) was tested with using cross-tabulation, $\chi^{2}$ and Cohen's Kappa estimates were determined. Intra-class correlation coefficient (ICC) 
analysis was performed to evaluate the inter-rater reliability between the participants. Each participant in the group evaluated every single photo separately; two-way random ICC was used. Bivariate Pearson correlation analysis was used to determine the correlation between participants' evaluation and participants' work experience.

\section{RESULTS}

The study included 40 patients with laryngeal lesions. The average age was 55.1 years $(\min 24-\max 76$ years). In the group, $10 \%$ of the patients were female and $90 \%$ were male.

Full survey responses were received from 30 otolaryngologists, with a mean age of 35.73 years (min 26-max 60 ) and a minimum of 2 and maximum of 35 years' work experience (mean: 10,56).

\section{Relationship Between Vascularization Patterns and Histopathology}

Histopathology revealed benign lesions in $27.5 \%$, malignant lesions in $40 \%$, and dysplastic changes in $32.5 \%$ of the cases. The patients lacking detectable vasculatures (\%7.5) included one who presented with a mass of granulation tissue covered with exudative material, while the other two presented with leukoplakia on a low-grade dysplastic lesion (Figure-1). In the surveyed cases, $29.7 \%$ were benign, $2.7 \%$ were dysplastic, and $43.2 \%$ were malignant.

Of the 37 lesions, 9 were classified as longitudinal and 28 as perpendicular by the authors. The distribution of the pathological lesions across the vascular patterns is shown in Table 1. The pathological diagnoses of the lesions associated with longitudinal vascular pattern were as follows: $8 / 9$ were benign, as five polyps (Figure-2), and three Reinke's edema, and $1 / 9$ was a low-grade dysplasia. The pathological diagnoses of the lesions associated with perpendicular vascular pattern were as follows: $16 / 28$ were malignant, $9 / 28$ were with high-grade dysplasia (Figure-3) and 3/28 were benign. The distribution of vascular patterns in benign, dysplastic and malignant conditions is shown in Table 2. In the benign cases, the longitudinal pattern was present in $72.7 \%$ and the perpendicular pattern was present in $27.3 \%$ of the lesions. In dysplastic and malignant lesions, the longitudinal pattern was observed in $3.8 \%$ (Figure-4), whereas $96.2 \%$ presented with perpendicular vascular pattern. The benign lesions with perpendicular vascular patterns (Figure-4), included a granular cell tumor and another two that were granulation tissue after laser cordectomy; the granulation here was not associated with fibrin accumulation. In dysplastic and malignant lesions, a low-grade dysplasia was evident with the longitudinal vascular pattern. The relationship between the vascularization pattern and the histopathological diagnosis was statistically significant.

The sensitivity (SE) and specificity (SP) of Image1 S endoscopy were 96\% (95\% CI 80-99) and $72 \%$ (95\% CI 39-93) respectively, for the differentiation of malignant and dysplastic lesions from benign lesions. The positive predictive value was $89 \%$ (95\% CI 75-95) and the negative predictive value was $88 \%$ (95\% CI 53-98) with the accuracy rate $89 \%$ (95\% CI 74-96) (Table-2).

\section{Feasibility of Using the Image1 S Enhancement System}

A total of 1110 responses were obtained from 30 participants after they observed the paired images in 37 respective cases. A cross tabulation analysis of the two categorical variables was performed for comparison of the qualitative evaluation results of the participants and the authors. The analysis revealed that the qualitative evaluation of the participants and the authors were significantly close to each other $(\chi 2[1]=462.92)$. The Cohen's kappa value was 0.63 , indicating a substantial agreement between the qualitative evaluations by the participants and the authors. The results showed $96.2 \%$ congruence between the participants' and the authors' observations with respect to the perpendicular lesions, and $61.2 \%$ congruence with respect to the longitudinal lesions. Inter-rater reliability between participants was tested by ICC, and the results of the test showed significant reliability of the participant observation data $(\operatorname{ICC}(2,30)=0.96,95 \%$ CI $[0.94$, $0.98])$.

The correlation between the participants' accuracy and their years of work was non-significant $(\mathrm{r}=0.18, \mathrm{p}=$ 0.331), thus, previous work (clinical) experience had no effect on the qualitative evaluation. 


\section{White Light versus Spectral Modes}

In their evaluation of the vascular patterns, the participants chose the most informative light mode between the white mode and the S modes. The survey showed that, in all cases, the spectral modes were preferred, on average, by $81.9 \%$ of the participants; white light was preferred on average by only $3.94 \%$. No mode preference was indicated, on average, by $14.59 \%$ of the participants.

\section{DISCUSSION}

Image enhancement systems increase the contrast of the blood vessels for improved visibility of the submucosal vasculatures. Using the NBI enhancement system, the ELS classified the submucosal vascular patterns in laryngeal lesions as longitudinal and perpendicular, with an association of the perpendicular pattern with malignancy, and the longitudinal pattern with benign conditions. In the present study, the vascular patterns reproduced by Image1 S endoscopy in benign and malignant lesions were highly consistent with those defined in the ELS classification. The prediction of the pathological diagnosis strongly correlated with the definitive pathological diagnosis of the lesions. Moreover, the evaluations of the patterns were highly consistent among the evaluators, irrespective of their work history, or their previous experience with image enhancement systems. These consistencies stated above, are directly related to improved visibility of the vasculatures by contrast enhancement. Therefore, Image1 $\mathrm{S}$ is considered to be an effective and practical technique for evaluation of the vascular characteristics of laryngeal lesions to predict malignancy.

Stanikova et al. used both NBI and the Image1 S system to investigate the relationship between the vascular patterns and the pathological diagnosis of laryngeal lesions (7). They found the sensitivity and specificity values for NBI endoscopy of $83 \%$ and $98 \%$ respectively, whereas those values for Image $1 \mathrm{~S}$ were $86 \%$ and $96 \%$, respectively. In diagnostic use, the high specificity of the test indicated a strong association of benign lesions with the longitudinal pattern and a weak association with the perpendicular pattern, suggesting an ability to detect the patterns in benign lesions. However, in our study, Image 1 endoscopy showed a higher sensitivity $(96 \%)$ and a lower specificity (72\%) values, compared to the previous study. In diagnostic use, the high sensitivity of the test is related to the improved detection of vascular patterns in dysplastic and malignant lesions, and it indicates a strong association between the perpendicular pattern and malignant lesions and a weak association between the longitudinal pattern and malignancy. Thus, malignant and dysplastic lesions were predicted with certainty with the Image1 S technique and, as a result, relatively few cases of cancer were missed in the endoscopic examination. Our study was conducted on a relatively small number of subjects; however, the sample size was sufficient because malignant and premalignant subjects were most prevalent in the whole sample. In addition, a highly sensitive test with many true positives requires a minimum sample size for statistically significant results. Image1 S endoscopy, therefore, proved to be a sensitive and reliable screening test for the prediction of malignancy in suspicious lesions presenting with a perpendicular vascular pattern.

The false positive and false negative cases and the cases excluded from the survey did not have any significant effect on the statistical parameters, but they are clinically important when screening to predict malignancy. The only false negative case was a low-grade dysplasia associated with a longitudinal pattern; this case may reflect the small extent of neo-angiogenesis in low grade dysplastic lesions (8). Among the false positive cases, one case with benign granular cell tumor and two cases with granulation tissue, were negatively associated with a perpendicular vascularization pattern. A perpendicular pattern in benign tumors has also been reported by Arens et al. for HPV-related papilloma. That study used contact endoscopy with NBI system and emphasized the differences in turning angle of the vessels within the vascular loop; where wide long turns in the papilloma differed from short, acute turns in terms of carcinogenesis (2). Distinction of these differences was not possible in our endoscopic examination, but coupling the image1 S system with contact endoscopy, which would result in a nearly 60 -fold gain in magnification, may help to clarify these distinctions. Two other false positive cases with granulation tissue after laser surgery appeared to be related to the wound healing processes, which can make vascular evaluation difficult.

The cases excluded from the survey were those presenting with leukoplakia and granulation tissue, and they 
featured with surface characteristics that limited the visualization of the vasculatures. The limitation in the leukoplakia case was the umbrella effect of keratosis (9). Likewise, the vasculatures in the granulation tissue cases were obscured due to the exudative material that covered the surface. For these reasons, endoscopic image enhancement techniques may have a limited value in keratotic and exudative lesions. Nevertheless, a high rate of accuracy with many true positives and true negatives was achieved in our study using Image1 $\mathrm{S}$ enhancement, apart from the few exceptions mentioned above.

This study also evaluated the practical utility of the ELS concept of vascular patterns, by conducting an electronic survey, on a group of otolaryngologists with a varying degrees of work experience. This part of the study had two major limitations: one was that digital images provide less information than actual video-endoscopic examination, and the other was the varying degree of knowledge among the evaluators regarding the ELS classification is variable. Despite these limitations, the survey results were consistent between the authors and the participants regardless of previous work experience and quality of the images, i.e., the vascular patterns were identified with high accuracy by all the evaluators. This study confirms the feasibility of using the ELS classification in routine endoscopic examination and for e-screening and e-learning purposes.

In the survey, we also questioned whether the spectral light images are preferred over the white light images for discrimination of the vascular patterns. Technically, white light provides sharpness and local brightness, whereas spectral modes provide the highest image contrast. The spectral modes have been used previously in several situations, including urinary bladder imaging, for improved visualization of the parathyroid gland in endoscopic thyroidectomy, during endonasal sinus surgery, and for treatment of oral cavity and oropharynx lesions in combination with contact endoscopy (10-13). Based on the empirical observations of the users in the present study, the spectral modes, which produced high contrast images, were apparently preferred over white light when examining laryngeal lesions.

\section{CONCLUSION}

Image1 S endoscopic enhancement system with spectral light modes provides an improved visibility of the vascular patterns defined by the ELS in laryngeal pathologies. A group of otolaryngologists with varying degrees of clinical expertise were able to use Image1 S technology to discriminate between perpendicular and longitudinal vascular patterns with accuracy and consistency. This study demonstrated the usefulness and feasibility of Image1 S endoscopic enhancement system to predict the diagnosis from vascular changes in laryngeal lesions.

\section{Data Availability Statement}

The datasets generated during and/or analyzed during the current study are available from the corresponding author on reasonable request.

\section{REFERENCES}

1. Muto M, Horimatsu T, Ezoe Y, Hori K, Yukawa Y, Morita S, et al. Narrow-band imaging of the gastrointestinal tract. J Gastroenterol. 2009;44(1):13-25.

2. Arens C, Piazza C, Andrea M, Dikkers FG, Tjon Pian Gi RE, Voigt-Zimmermann S, et al. Proposal for a descriptive guideline of vascular changes in lesions of the vocal folds by the committee on endoscopic laryngeal imaging of the European Laryngological Society. Eur Arch Otorhinolaryngol. 2016;273(5):1207-14.

3. Kamphuis GM, de Bruin DM, Brandt MJ, Knoll T, Conort P, Lapini A, et al. Comparing Image Perception of Bladder Tumors in Four Different Storz Professional Image Enhancement System Modalities Using the iSPIES App. J Endourol. 2016;30(5):602-8.

4. Puxeddu R, Sionis S, Gerosa C, Carta F. Enhanced contact endoscopy for the detection of neoangiogenesis in tumors of the larynx and hypopharynx. Laryngoscope. 2015;125(7):1600-6. 
5. Emiliani E, Talso M, Baghdadi M, Barreiro A, Orosa A, Servian P, et al. Evaluation of the Spies (TM) modalities image quality. Int Braz J Urol. 2017;43(3):476-80.

6. Adel K. El-Naggar JKCC, Jennifer R. Grandis, Takashi Takata, Pieter J. Slootweg. WHO Classification of Head and Neck Tumours. 4 ed. Lyon, France: IARC Press; 2017.

7. Stanikova L, Walderova R, Jancatova D, Formanek M, Zelenik K, Kominek P. Comparison of narrow band imaging and the Storz Professional Image Enhancement System for detection of laryngeal and hypopharyngeal pathologies. Eur Arch Otorhinolaryngol. 2018;275(7):1819-25.

8. Rzepakowska A, Zurek M, Grzybowski J, Pihowicz P, Gornicka B, Osuch-Wojcikiewicz E, et al. Correlation of narrow band imaging vascular patterns with immunohistological microvessel density in vocal fold lesions. Braz J Otorhinolaryngol. 2019.

9. Arens C, Glanz H, Dreyer T, Malzahn K. Compact endoscopy of the larynx. Ann Otol Rhinol Laryngol. 2003;112(2):113-9.

10. Carta F, Sionis S, Cocco D, Gerosa C, Ferreli C, Puxeddu R. Enhanced contact endoscopy for the assessment of the neoangiogenetic changes in precancerous and cancerous lesions of the oral cavity and oropharynx. Eur Arch Otorhinolaryngol. 2016;273(7):1895-903.

11. Lin P, Han P, Liang F, Cai Q, Chen R, Yu S, et al. Characteristics of the parathyroid gland in endoscopic thyroidectomy with the application of an image enhancement system. Surg Endosc. 2018;32(9):3925-35.

12. Lopez A, Liao JC. Emerging endoscopic imaging technologies for bladder cancer detection. Curr Urol Rep. 2014;15(5):406.

13. Mulazimoglu S, Anadolu Y, Beton S, Basak H, Yorulmaz I, Meco C. Preference and Perception of Enhanced Images During Endonasal Endoscopic Surgery. J Craniofac Surg. 2018;29(8):2296-8.

TABLES

Table 1 Distribution of vascular patterns in the benign, dysplastic and malignant lesions

\begin{tabular}{lllll}
\hline & Overall $(\mathrm{N}=37)$ & Benign $(\mathrm{N}=11)$ & Dysplasia $(\mathrm{N}=10)$ & $\begin{array}{l}\text { Malignant } \\
(\mathrm{N}=16)\end{array}$ \\
\hline Perpendicular & 28 & $310.7 \%$ & $932.1 \%$ & $1657.2 \%$ \\
Longitudinal & 9 & $888.9 \%$ & $111.1 \%$ & $00 \%$ \\
$\chi^{2}(2)=20.30, \mathrm{p}$ & $\chi^{2}(2)=20.30, \mathrm{p}$ & $\chi^{2}(2)=20.30, \mathrm{p}$ & $\chi^{2}(2)=20.30, \mathrm{p}$ & $\chi^{2}(2)=20.30, \mathrm{p}$ \\
$<0,001$ & $<0,001$ & $<0,001$ & $<0,001$ & $<0,001$ \\
\hline
\end{tabular}

Table 2 Two-by-two contingency table for diagnostic test results

\begin{tabular}{llll}
\hline Image1 S observation & Malign or dysplastic & Benign & Totals \\
\hline Perpendicular Pattern & $25(96.2 \%)$ & $3(27.3 \%)$ & 28 \\
& $\begin{array}{l}\text { (True-positive) } \\
\text { Longitudinal Pattern }\end{array}$ & $\begin{array}{l}\text { (False-positive) } \\
(3.8 \%)\end{array}$ & 9 \\
Total & 26 & $(72.7 \%)$ & 9 \\
\hline
\end{tabular}

\section{FIGURE LEGENDS}

\section{Figure 1. Endoscopic views of the lesions lacking detectable vasculatures}

Top left and right images demonstrate a left sided vocal fold granuloma in spectra B (S-B) and spectra A 
(S-A) modes, respectively (patient \#38 in the survey). The surface of the lesion is covered with a blanket of fibrin-like material, showing no identifiable vascularization. Bottom images show S-B and S-A view of a keratotic leukoplakia with low-grade dysplasia on the left vocal cord (patient \#39), vascular patterns are obscured by the umbrella effect of the plaque.

\section{Figure 2. Longitudinal vascular pattern}

Side by side view of $\mathrm{W}$ and S-B images of the vocal cords in the patient \#22. Note the meandering tortuous vessels on a polyp on the left vocal cord (asterisk), and a small polyp with ectatic vessels running parallel to the right vocal cord (arrows). These changes are classified as longitudinal.

\section{Figure 3. Perpendicular vascular pattern}

Side by side view of $\mathrm{W}$ and S-A images of the vocal cords in the patient \#35. The right cord is thick, with an irregular surface, and associated with subglottic edema; diagnosed as high-grade dysplasia. S-A image shows perpendicular vessel loops (arrows) as yellow-greyish spots, indicating the presence of a malignant pathology. On the $\mathrm{W}$ image, the vascular pattern is unclear.

\section{Figure 4. The false-negative and false-positive cases}

Top: Side by side view of $\mathrm{W}$ and S-A images of a keratotic lesion on the left vocal cord, patient \#14. Dilated and tortuous vessels around the white keratotic patch (arrows) are classified as longitudinal (false-negative) although the lesion truly is a low-grade dysplasia.

Central: W and S-A images of a granulation tissue on the left vocal cord, occurred 3 months after laser cordectomy (patient \#1). The circled area contains worm like vessels with small sharp turns, lacking a longitudinal orientation, and were classified improperly as perpendicular (false-positive).

Bottom: W and S-A images of a benign granular cell tumor on the left vocal cord (patient \#4). Worm like vessels (arrows) were classified as perpendicular (false-positive).

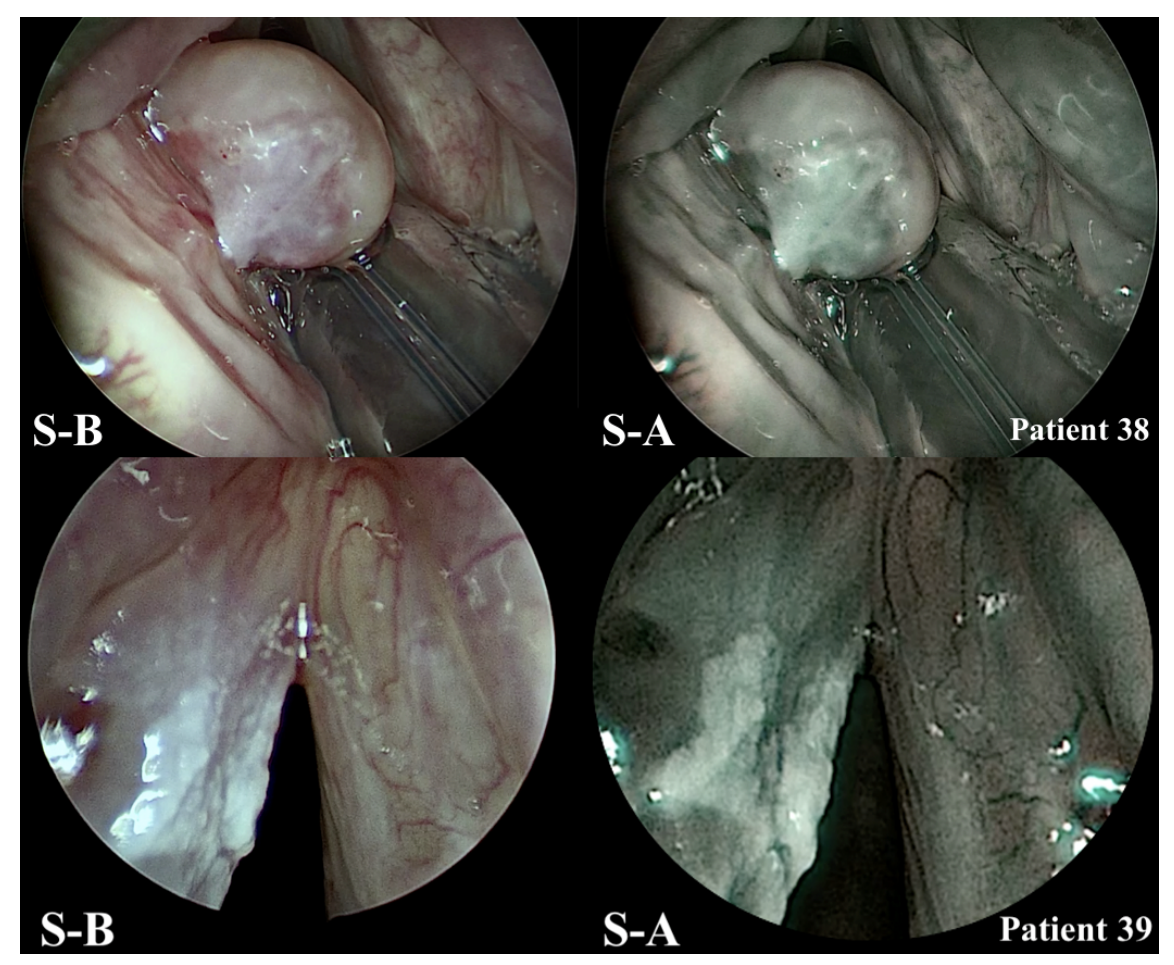



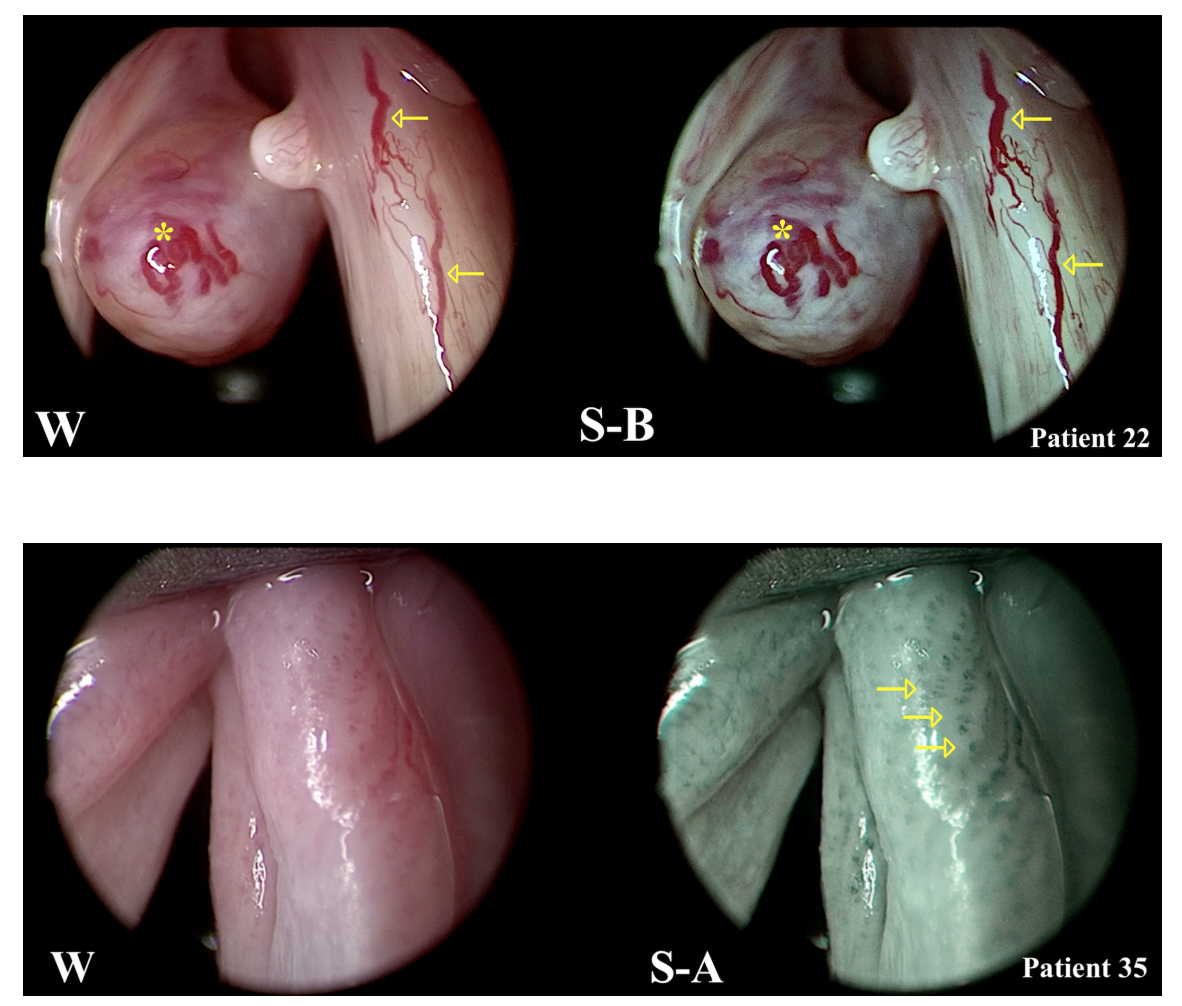


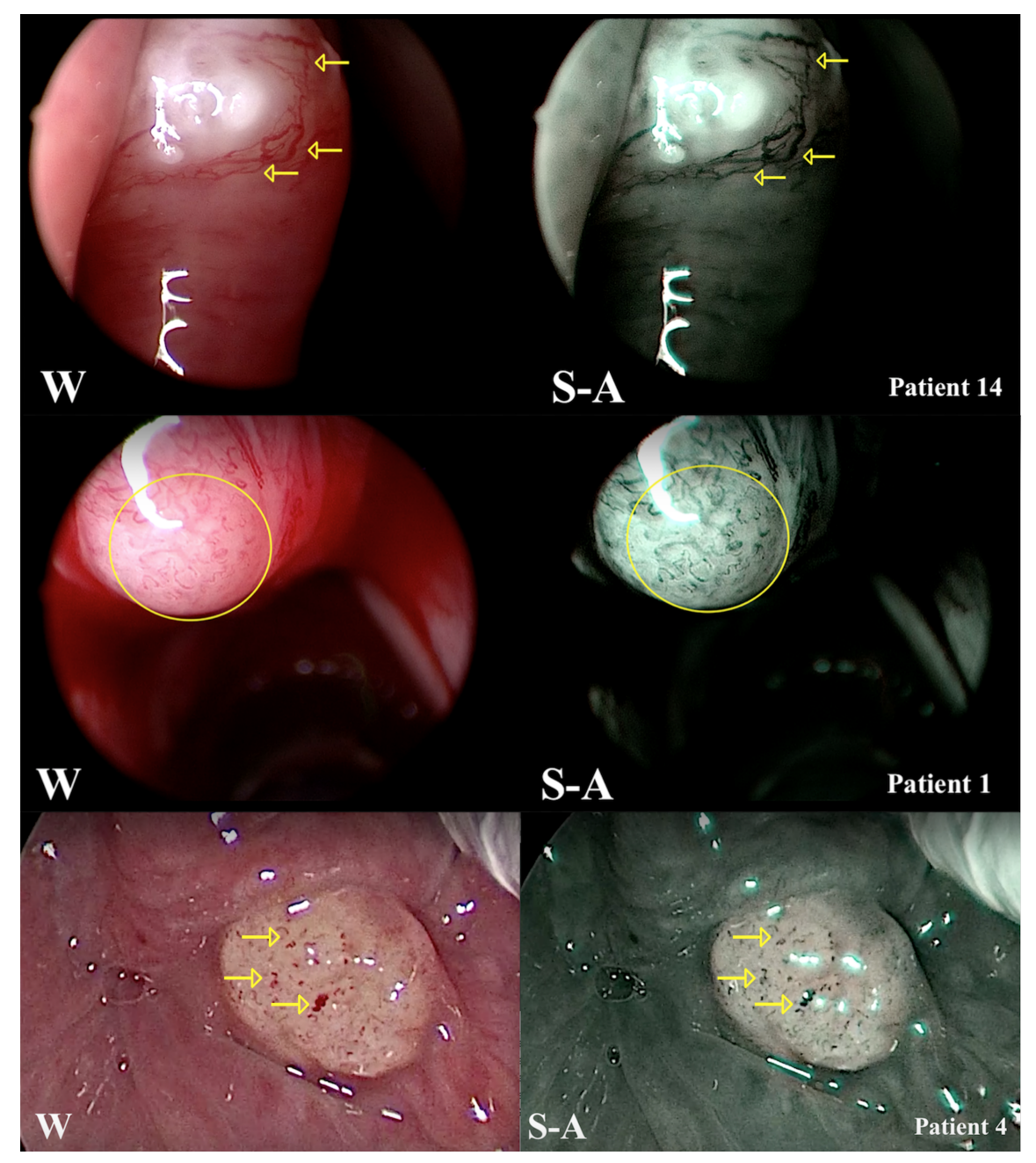

